\title{
Gewänder als Parerga. Zu Herders „Plastik"
}

Natalie Binczek ${ }^{*}$

\begin{abstract}
This article analyses J. G. Herder's essay on sculpture, particularly the relation between seeing and feeling, outside and inside, surface and body, painting and sculpture in aesthetic theory in the second half of the $18^{\text {th }}$ century. Herder's intention is the foundation of autonomous sculptural art upon the physiology of touch, not performed, however, through the act of touching, but through visual imitation, by observing the statue. In this sense, the distinctiveness of sculpture would not only be the compactness of the body but the tense relationship between compactness and its articulation, the accessories. In the tension between work and accessories - erga and parerga -, the clothes of statues constitute a "dead" addition, that disturbs the effect of the "living" body. The "wet" clothes of antique statues, on the other hand, would be so "transparent", that they would appear as a second skin. Parerga of this kind would not represent something superfluous and inconvenient, but rather a necessary element of sculptural art, by covering - yet not completely denying - the organic interior that refers to death.
\end{abstract}

Keywords: Herder; Kant; Aesthetics; Sculpture.

Zusammenfassung: Der Essay analysiert den Aufsatz über die Plastik von J. G. Herder im Hinblick auf das Verhältnis von Sehen und Fühlen, Außen und Innen, Oberfläche und Körper, Malerei und Skulptur in der ästhetischen Theorie der zweiten Hälfte des 18. Jahrhunderts. Herders Intention ist die Begründung einer autonomen Bildhauerkunst aus der Physiologie des Tastsinnes, der jedoch nicht in Form des Berührens realisiert wird, sondern im visuellen Nachvollzug beim Betrachten der Statue. Charakteristikum der Plastik wäre dem-

Die Autorin ist wissenschaftliche Assistentin im Fach Germanistik und Allgemeine Literaturwissenschaft an der Universität Siegen. 
Stichwörter: Herder; Kant; Ästhetik; Plastik.

Resumo: $\mathrm{O}$ artigo analisa o ensaio de J. G. Herder sobre escultura, particularmente a relação entre ver e sentir, exterior e interior, superfície e corpo, pintura e escultura na teoria estética da segunda metade do século XVIII. A intenção de Herder é a fundação de uma arte escultural autônoma a partir da fisiologia do tato, não realizada, contudo, através do ato de tocar, mas através da imitação visual ao se observar a estátua. Neste sentido, a distintividade da escultura não estaria apenas na natureza compacta do corpo, mas na tensa relação entre o compacto e sua articulação, os acessórios. Na tensão entre o trabalho e os acessórios - erga e parerga -, as roupas das estátuas constituem uma adição "morta", que perturba o efeito do corpo "vivo". As roupas "molhadas" das estátuas da antiguidade, por outro lado, seriam tão "transparentes" que pareceriam uma segunda pele. Esse tipo de parerga não representa algo supérfluo ou inconveniente, mas sim um elemento necessário da arte da escultura, cobrindo - mas não completamente negando - o interior orgânico que faz referência à morte.

Palavras-chave: Herder; Kant; estética; escultura.

\section{Sehen und Tasten}

Herders 1778 in der zweiten Fassung erschienene „Plastik“verhandelt eine ästhesiologische Gattungstypologie der Bildhauerkunst und ihres perzeptiven Mediums, des Tastsinns. Danach gilt die Körperwahrnehmung als alleiniges Privileg des ,tastenden Gefühls', wie dementsprechend die Körperdarstellung alleiniges Privileg der Bildhauerkunst ist. Jedoch stößt 
diese Festlegung bereits im Anschluss an ihre erste Formulierung auf Widersprüche. So lässt sich die Abgrenzung von der Malerei und ihrem Rezeptionsmedium, dem Sehen, kaum aufrecht erhalten, wie bereits zu Anfang dieser Schrift deutlich wird.

Kommt in die Spielkammer des Kindes, und sehet, wie der kleine Erfahrungsmensch fasset, greift, nimmt, wägt, tastet, mißt mit Händen und Füßen, um sich überall die [...] ersten und notwendigsten Begriffe von Körpern [...] zu verschaffen (Herder: 249).

Das Kind macht seine ersten Erfahrungen haptisch, ,mit Händen und FüBen'. Auf diese Weise bildet es die ,Begriffe von Körpern“ heraus und schafft so die Voraussetzung, welche es zur Wahrnehmung von plastischen Kunstwerken befähigen wird. Zugleich lässt sich aber an der entwicklungsphysiologischen Perspektive ablesen, weshalb haptische und visuelle Eindrücke einander derart überlagern, dass sie letztlich - auch in der ästhetischen Rezeption - nicht voneinander zu lösen sind. Die Wahrnehmungen des Auges und der Hände unterscheiden sich zwar, ihre Verknüpfung bereits im frühesten Kindesalter hingegen macht die Unterschiede nicht mehr nachvollziehbar. „Nur da wir von Kindheit auf unsre Sinne in Gemeinschaft und Verbindung brauchen: so verschlingen sich alle, insonderheit der gründlichste und der deutlichste der Sinne, Gefübl und Gesicht.“ (250) Ihre, verschlungene', Gemeinschaft und Verbindung' muss erst wieder aufgelöst werden, um die jeweiligen Funktionsbereiche zu differenzieren; also das zu leisten, was sich der Text selbst aufgibt.

Wenn Herder nun eine Unterscheidung der Kunstgattungen in Korrespondenz zu den Sinneswahrnehmungen entwirft, muss er bei der Bestimmung der Bildhauerkunst in jenes - allerdings kaum rekonstruierbare Entwicklungsstadium zurückzukehren versuchen, in welchem die Bereiche des Gefühls und Gesichts noch voneinander abgegrenzt waren. Um eine Freilegung der haptischen Wahrnehmungsqualität muss eine solche Ästhetik der Bildhauerkunst bemüht sein. Jedoch plädiert die von Herder entfaltete haptische Ästhetik für keine Rückwendung zum sensorischen Ausgangspunkt. Sie setzt vielmehr supplementär an, indem sie an der Vermischung der Empfindungen festhält und unter dieser Voraussetzung das 
Sehen auf die Qualität des Tastsinns zu wenden versucht. Insofern wird die als, Verschlingung' bezeichnete Beziehung zwischen beiden Sinnen noch einmal verschlungen. Anstatt die Operation des ,tastenden Gefühls' in Reinform einzufordern, entwickelt Herder das Konzept einer haptischen Führung des Gesichtssinns. „Seht jenen Liebhaber“, heißt es dementsprechend, „der tiefgesenkt um die Bildsäule wanket. Was tut er nicht, um sein Gefühl zum Gesicht zu machen, zu schauen als ob er im Dunkeln taste?"“ (254)

Auf dem kursiv gesetzten, schauen' liegt der Akzent dieses Satzes; ein ,schauen', welches konditional, ,als ob' es sich um ein ,Tasten' handle, bestimmt wird. Die Fiktion einer Tastempfindung entsteht. Zumindest in der Perspektive des Liebhabers erweist sich die ästhetische Koppelung von Skulptur und Haptik als eine Als-ob-Konstruktion, die nur mit Hilfe des Auges und großer Anstrengung erlangt werden kann. Fast scheint es, als müsse sie unentwegt aufs neue aktualisiert werden. „Darum gleitet er: sein Auge ward Hand, der Lichtstrahl Finger, oder vielmehr seine Seele hat einen noch viel feinern Finger als Hand und Lichtstrahl ist, das Bild aus des Urhebers Arm und Seele in sich zu fassen" (254). Herder erzählt hier die Geschichte einer ästhetisch ausgelösten Vertauschung bzw. Täuschung der Sinneseindrücke, deren Ort die Seele ist. Er zeichnet eine Metamorphose nach, welche aus dem Auge eine Hand macht und aus dem Lichtstrahl einen Finger. „Sie hats!“ exklamiert der Text weiter und beschreibt damit eine Klimax, die nicht von Dauer ist, sondern sich allmählich wieder in die konditionale Rede zurückverwandelt: „Die Täuschung ist geschehn: es lebt, und sie fühlt, daß es lebe; und nun spricht sie, nicht, als ob sie sehe, sondern taste, fühle" (Herder 254). In dem Augenblick, in welchem das ,Bild' zu einer lebenden Statue wird, spricht die Seele des Liebhabers als würde sie ,tasten' und ,fühlen'. Eine programmatische Wendung findet statt. Denn nachdem der Text mit dem Ausruf des ,Sie hats!' die Erregung des Ereignisses rhetorisch vollzieht, kehrt er grammatisch in der Konditionalaussage am Ende dieser Metamorphose zum Anfangspunkt zurück, um nun im Rekurs auf den Liebhaber vielleicht auch seinen eigenen Rededuktus zu thematisieren. ${ }^{1}$

1 Der Pygmalion-Bezug, der immerhin auch im Untertitel ausgestellt wird, erweist sich als eine Täuschung. 


\section{Malerei und Plastik}

Der Sinn des Gesichts würkt flach, er spielt und gleitet auf der Oberfläche mit Bild und Farbe umher; überdem hat er so Vieles und so Zusammengesetztes vor sich, daß man mit ihm wohl nie auf den Grund kommen wird. Er borgt von andern und baut auf andre Sinne: ihre Hülfsbegriffe müssen ihm Grundlage sein, die er nur mit Licht umglänzet (253).

Was das Auge wahrnimmt, ist derart ,zusammengesetzt ${ }^{6}$ und - wie wohl ergänzt werden kann - vielgestaltig, dass es zur Herstellung vollständiger Eindrücke auf die Hilfe, anderer Sinne', vor allem aber des Tastsinns, angewiesen ist, von dem es seine Begriffe ,borgt'. Die hier als allgemeingültiges Gesetz formulierte Hilfsbedürftigkeit des Gesichtssinns kontrastiert aber dem zuvor geschilderten ästhetischen Rezeptionsentwurf, indem dieser die Entstehung haptischer Eindrücke gerade umgekehrt auf der operativen Grundlage des Sehens vollzieht. In dieser Hinsicht zumal ist der Tastsinn vom Gesichtssinn abhängig. Die fühlbare Qualität einer Plastik teilt sich dem Auge mit.

Wie jedoch verhält es sich vor dem Hintergrund dieser, verschlungenen' Differenzierung beider Sinne mit den ihnen jeweils zugeordneten und strukturell an sie angelehnten Künsten?

Ich verfolgte beide Künste und fand, daß kein einziges Gesetr, keine Bemerkung, keine Würkung der Einen, ohn Unterschied und Einschränkung auf die andre passe. Ich fand, daß gerade je eigner Etwas Einer Kunst sei und gleichsam als einheimisch derselben in ihr große Würkung tue, desto weniger lasse es sich platt anwenden und übertragen, ohne die entsetzlichste Würkung (256).

Deutlich setzt Herder auf eine undurchlässige Demarkationslinie zwischen der Bildhauerei und Malerei. ,Kein einziges Gesetz', hebt er hervor, ließe sich aufgrund der ihnen jeweils, eigenen' Selbstreferenz gleichermaßen auf beide Künste anwenden. ${ }^{2}$ So grundlegend wird der Un-

2 Der Sinnesdiskurs hat demgegenüber immer wieder auf die Kompatibilität zwischen dem Tast- und dem Gesichtssinn aufmerksam gemacht. Dass sich 
terschied zwischen ihnen aufgefasst, dass keine ,Bemerkung' oder, Wirkung' gleich bliebe, würde sie von der einen in die andere übertragen werden. Damit ist eine der Autonomie der Kunst entsprechende autonome Konstitution der Kunstgattungen etabliert. Wie Kunst von NichtKunst geschieden wird, so auch innerhalb der ersten die einzelnen Kunstgattungen. ${ }^{3}$ Ein zentrales Anliegen der „Plastik“ besteht daher im Einkreisen dessen, was den Werken der Bildhauerei ,eigen', was nur in ihnen, einheimisch“ ist. Nach diesem Schema erfolgt auch die Unterscheidung der den Künsten jeweils eigenen Organisationsformen. So kennzeichnet die Verteilung im „Nebeneinander“ die Malerei, wohingegen die Plastik als eine Kunst bestimmt wird, deren „Teile auf einmal in-nebenbei einander" (257) strukturiert sind: sowohl ineinander enthalten, womit auf Kompaktheit und Solidität im Aufbau der Elemente verwiesen wird, als auch ,neben-und beieinander', womit eine gleichsam ,para'-artige ${ }^{4}$ Umgebung in den Zusammenhang eingebunden wird. Zudem ist im ,Nebeneinander' eine Überschneidung mit der Organisationsform der Malerei gegeben. Wird diese deshalb von der Plastik einverleibt, kann sie als eines ihrer Bestandteile aufgefasst werden und liegt darin ein Widerspruch zum Grundsatz der Autonomie der beiden Kunstarten vor? Und in der Tat scheint sich die Grenze zwischen ihnen einerseits ins Innere der Bildhauerei zu verschieben. Andererseits aber drückt sich in den Präpositionen des ,Neben- und Beieinander', des ,Para'-artigen dieser Bezeichnungen, zugleich auch eine Dezentrierung der plastischen Form selbst aus,indem sich ihre geschlossene Dichte auf Neben- und Beigeordnetes, auf das von ihr Unterschiedene, hin öffnet. Dieses Argument ist von zentraler Bedeutung für die weiteren Ausführungen, denn es formuliert bereits auf der Ebene der elementaren Organisation eine Span-

Herder eingangs bereits auf Diderot bezieht, hängt damit zusammen, dass er die Analogie mit Abweichung aufgebrochen und zugunsten einer starken Differenz umgewertet hat.

3 An dieser Stelle setzt Herder das Anliegen Lessings im „Laokoon“ fort und damit die Ablehnung einer an dem „ut-pictura-poesis“-Diktum orientierten Poetik bzw. Ästhetik.

4 Vgl. dazu Genette. 
nung, ${ }^{5}$ welche der Text, wie ich zu zeigen versuchen möchte, in seinem weiteren Verlauf ästhetisch als Gleichzeitigkeit von ,Ergon` und ,Parergon` der Plastik ausfaltet.

\section{3. Ästhetische Autonomie und Gewänder}

Nachdem das erste Kapitel eine Theorie der Sinne sowie der ihnen zugeordneten Künste in allgemeinen Zügen umrissen hat, setzt das zweite konkreter an. Es widmet sich der Erfassung einzelner antiker Skulpturen und versucht sie dabei nach den zuvor erarbeiteten Kategorien zu beschreiben. Eingeleitet wird dieses Vorgehen von einer als Frage formulierten Überschrift: „Bildhauerkunst und Malerei, warum bekleiden sie nicht mit Einem Glücke, nicht auf Einerlei Art?“ (259). „Antwort“, so der Anfang des folgenden Abschnitts: „Weil die Bildnerei eigentlich gar nicht bekleiden kann und die Malerei immer kleidet“ (259). Die deskriptive Annäherung an einzelne Plastiken erfolgt über die Kleider. Dabei weist dieser Begriff einen konnotativen Resonanzraum auf, der auch die Rhetorik des Ornaments und mit ihr die schmuckvolle Ausgestaltung einer Rede einbezieht. Hier aber richtet es sich zunächst ganz buchstäblich auf das von der Ästhetik des 18. Jahrhunderts durchaus viel diskutierte Problem der plastischen Gewänderdarstellung. ${ }^{6}$ Was die Zwischenüberschrift als Defizit zur Sprache kommen lässt, nämlich die Unfähigkeit der Bildhauerkunst ,zu bekleiden', wird der Text im Folgenden zum uneinholbaren Vorsprung gegenüber der Malerei erklären. Aus der vermeintlichen Unfähigkeit wird eine ästhetische Ressource. Wie aber begründet Herder diese Aussage? Die Bildhauerei „kann gar nicht bekleiden“, so seine Antwort, „denn offenbar verhüllet sie gleich unter dem Kleide, es ist nicht mehr ein menschlicher Körper, sondern ein langgekleideter Block“ (260). Anstatt sich über den Körper zu

5 „Bildnerei schafft schöne Formen, sie drängt in einander und stellt dar, notwendig muß sie also schaffen, was ihre Darstellung verdient, und was für sich da steht. Sie kann nicht durch das Nebeneinander gewinnen, daß Eins dem Andern aushelfe und doch also Alles so schlecht nicht sei: denn in ihr ist Eins Alles und Alles nur Eins“ (258).

6 Winckelmann, Lessing etc. 
号 legen, setzt sich das Kleid - ,offenbar ${ }^{6}$ - an dessen Stelle und deformiert

$\stackrel{N}{N}$ ihn. Eine Deformation, die geradezu auf die Herstellung plastischer Werke

Z zurückverweist, klingt doch im ,Block' das unbearbeitete Material, der Mar-

i mor- oder Steinblock, an. Keine Blöcke, sondern ,Körper und Formen',

I wie an anderer Stelle bereits festgelegt und mehrfach wiederholt, sind die

Gegenstände der Bildhauerei, will sie den Anforderungen ihrer ästhetischen

$\square$ Eigengesetzlichkeit gerecht werden. Ausschließlich die Darstellung des blo-

继 Ben, unbekleideten und deshalb auf seine organische Beschaffenheit reduzierten Körpers korrespondiert dem ,eigentlichen` Begriff, auf welchen die Malerei als Kunst der Bekleidung, der Verhüllung und damit des Sekundären, Hinzugefügten und Abgeleiteten gänzlich verzichten muss.

„Und nun in der Kunst ist ein Gewand von Stein, Erz, Holz ja im höchsten Grade drückend!“ (260). Das Material, dessen sich die Bildhauerei bedient, eignet sich nicht zur Darstellung von Gewändern oder Kleidern. „Es ist kein Schatte, kein Schleier, gar kein Gewand mehr: es ist ein Fels voll Erhöhung und Vertiefung, ein herabhangender Klumpe“ (260). Das Material verfehlt die Weichheit und Durchlässigkeit gewebter Stoffe. Es verweist auf seine eigene feste Konsistenz als ,herabhängender Klumpen‘ und unterbricht so die Illusion einer Belebung, welcher die plastische Körperdarstellung gleichwohl aber ästhetisch verpflichtet ist.

„Tue die Augen zu und taste, so wirst du das Unding fïblen" (260). Die Unangemessenheit der Gewänderdarstellung in der Bildhauerei soll nun in der Beurteilung des dafür ausgewiesenen Wahrnehmungssinns spürbar werden. Mit geschlossenen Augen ist es dem apostrophierten Du aufgetragen, ,das Unding zu fühlen'. Anders als der ,Liebhaber', der trotz der Evokation des haptischen Eindrucks dennoch in körperlicher Distanz zur Statue bleibt und somit eine wichtige Regel ästhetischer Rezeption befolgt, wird hier - zum ersten Mal in diesem Text - zur unmittelbaren Kontaktaufnahme aufgerufen. Dennoch wird mit diesem Aufruf nicht gegen das Berührungsverbot der Kunst verstoßen, insofern das ,Unding', welches es dabei wahrzunehmen gilt, keinen ästhetischen Wert aufweist; ein misslungenes, ja überhaupt kein Kunstwerk ist. Müsste sich diese Erfahrung aber bei der Berührung der unbekleideten Körperpartien der Statue nicht wiederholen? Müsste nicht die gesamte Plastik ohne Unterstützung des Auges denselben haptischen Eindruck hervorrufen? Ein ,Unding ‘ühl- 
bar machen? Weshalb sollen der Arm oder die Brust, obwohl aus demselben Material gemacht wie das Gewand, von dieser Ernüchterung ausgenommen sein? Der Text gibt keine Antwort. Statt dessen fährt er fort:

Und man kann überhaupt den Grundsatz annehmen, „daß wo der Griechische Künstler auf Bildung und Darstellung eines schönen Körpers ausging, wo ihm nichts Religiöses und Charakteristisches im Wege stand, wo seine Figur ein freies Geschöpf der Muse, ein substanzielles Kunstbild, kein Emblem, keine historische Gruppe, sondern Bild der Schönheit sein sollte, da bekleidete er nie, da enthüllte er [...] (262).

Soll eine Skulptur zur Anschauung des autonom Schönen - ein ,substanzielles Kunstbild' - werden, darf sie ,nichts Religiösem und Charakteristischem'verpflichtet sein. Sie darf weder als ,Emblem' noch als, historisches‘ Dokument dienen, sondern muss, so der ,Grundsatz', schöne und unbekleidete Körper darstellen. Damit ist die Manifestation einer von jeder Zweckbestimmung freigesetzten Ästhetik konturiert.

Aber eine Gestalt der Schönheit, der Liebe, des Reizes, der Jugend, Bacchus und Apollo, Charis und Aphrodite, unter einem Mantel von Stein wäre Alles, was sie sind, was sie hier durch den Künstler sein sollen, verschleiert und verloren (262).

Bereits bis zu diesem Punkt bildet Herders Text einen komplexen argumentativen Zusammenhang. Er setzt die Bildhauerkunst autonom, indem er sie von religiösen, dokumentarischen sowie repräsentativen Zwecken freispricht und die Kleidung - als würde sie für diese Zwecke einstehen - abzulegen auffordert. Als über die autonome Ästhetik gestülpte Fremdreferenz müssen Kleider von der plastischen Darstellung eines menschlichen Körpers, dem Signifikanten des Schönen, entfernt werden. Das Postulat der Enthüllung korreliert demnach dem Postulat der ästhetischen Autonomie und Selbstbezüglichkeit, die sich jedoch ausschließlich, wie bereits hervorgehoben, dem haptisch geführten Auge mitteilt. Wo dieses nämlich die Hand nicht stützt, wo sie selbst tastet und fühlt, dort begegnet sie ,Undingen', ,Klumpen'. Die Haptik dient allein der Feststellung 


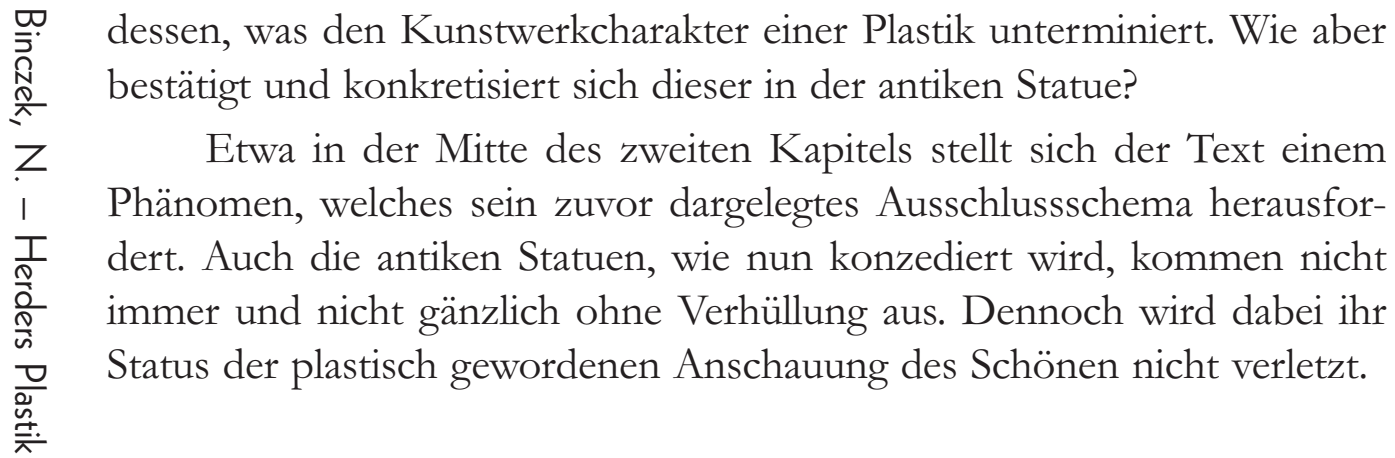

Wo auch der Grieche bekleiden mußte, wo es ihm ein Gesetz auflegte, den schönen Körper, den er bilden wollte, und den die Kunst allein bilden kann und soll, hinter Lumpen zu verstecken; gabs kein Mittel, dem fremden Drucke zu entkommen, oder sich mit ihm abzufinden? Zu bekleiden, daß doch nicht verbüllt würde? (263)

Ein paradoxer Ausweg wird hier erwogen: zu ,bekleiden', ohne zu ,verhüllen', der ein paar Zeilen weiter seine Auflösung erfährt: „Kurz, es sind der Griechen nasse Gewänder" (264). Wurde die Darstellungsmöglichkeit des schönen nackten Körpers unterbunden oder eingeschränkt, kannte der ,Grieche' dennoch eine Technik, wie er sowohl dieser Einschränkung als auch der ästhetischen Maßgabe gerecht werden konnte; wie er den ,fremden Druck' abzulegen hatte, ohne auf die Kleidung selbst zu verzichten, mithin eine Kleidung bildhauerisch zu ermöglichen, die den Körper weder ,drückt' noch ,verfremdet'. „Wie“, fragt Herder in diesem Zusammenhang, „wenn er [der Körper] durchschiene?“ (263). Zur Diskussion wird somit ein Gewänderkonzept gestellt, welches im ,Durchscheinen“ eine nach der Logik der Malerei erzeugte Sichtbarkeit des Körpers anspricht und deshalb umgehend wieder korrigiert wird:

In der Bildnerei [...] kann nichts durchscheinen: sie arbeitet für die Hand und nicht fürs Auge. Und siehe, eben für die Hand erfanden die feinen Griechen Auskunft. Ist nur der tastende Finger betrogen, daß er Gewand und zugleich Körper taste; der fremde Richter, das Auge, muß folgen (263 f.).

Nachdem sich diese Gleichung im Verlauf des Textes mehrfach relativiert hat, wird sie hier wieder festgelegt. Die Bildhauerei ,arbeitet für 
die Hand und nicht fürs Auge‘. Um dieser Prämisse zu genügen, müssen sich die Gewänder der Statuen als gleichsam eingeschmolzener Bestandteil des plastischen Körpers zu erkennen geben. Nur auf diese Weise können sie in die Täuschung seiner Vitalität einbezogen werden. Eine Täuschung, die vom Text selbst allerdings durch eine Bezugnahme auf den Gesichtssinn - , und siehe - gestört wird. Was indes zu ,sehen' sei, sind Gewänder, die dem Tastsinn eine ,Auskunft ' geben. Aber welche? Auch die haptischen Feststellungen des ,Steinklumpens' oder ,Undings' sind als Auskünfte etwa der Materialbeschaffenheit zu werten. Hier jedoch, dies macht der nächste Satz deutlich, soll dem ,tastenden Finger' eine trügerische Auskunft erteilt werden. Er soll den Eindruck gewinnen, daß er Gewand und zugleich Körper taste`. Ein zweifacher Betrug also. Die Paradoxie der nicht verhüllenden Kleider entspricht dabei dem haptischen Betrug, sowohl ein Gewand als auch einen lebenden Körper zu fühlen. Analog der an anderer Stelle beschriebenen Täuschung des ,Liebhabers', welcher allmählich die Statue sich ,verlebendigen' sieht, wird hier der Betrug einer Einheit des Körpers mit den ihn umhüllenden Gewändern hervorgerufen.

Es war nämlich einzige Auskunft, den tastenden Finger und das Auge, das jetzt nur als Finger tastet, zu betrügen: ihm ein Kleid zu geben, das doch nur gleichsam ein Kleid sei, Wolke, Schleier, Nebel - doch nein, nicht Wolke und Nebel, denn das Auge hat hier nichts zu nebeln; nasses Gewand gab er ihm, das der Finger durchfühle! (264)

Der Finger und das Auge werden nun gleichermaßen als Adressaten dieser ,Auskunft' bezeichnet. Ihre Funktionen haben sich einander angeglichen, wenn es hier vom Auge heißt, dass es ,jetzt nur als Finger taste'. Der Betrug beider Sinne ist gelungen. Er verdankt sich der Darstellung eines im ,gleichsam' eingeschränkten Kleides. Vom ,Unding' und ,Steinklumpen' bewegt sich der Text hier auf ein kursiv markiertes, Gleichsam` zu, mit welchem sprachlich eine Uneindeutigkeit, ein Abstand zwischen Gegenstand und Bezeichnung angezeigt wird. Die Rede ist nämlich von einem Kleid, das letztlich keines ist, es aber aufgrund dieser Selbstauflösung dem Finger ermöglicht, dass dieser ,durchfühle', so der Wortlaut; dass er sich sozusagen bis auf den Körper hindurchtaste. Jedoch „die Fülle der Körpers, die kein Gleichsam, die Wesen der Kunst ist, war und blieb Hauptwerk“ (264). 


\title{
4. Parergon
}

Zum ,Hauptwerk' treten die ,Nebenwerke', - in der Terminologie Kants - die ,Parerga' hinzu. Ausdrücklich werden in der Kritik der Urteilskraft Gewänder an Statuen dem parergonalen Zierat zugerechnet und als äußerliche „Zutat“ dem innerlichen „Bestandstück“ entgegengesetzt:

\begin{abstract}
Selbst was man Zieraten (Parerga) nennt, d.i. dasjenige, was nicht in die ganze Vorstellung des Gegenstandes als Bestandstück innerlich, sondern nur äußerlich als Zutat gehört und das Wohlgefallen des Geschmacks vergrößert, tut dieses doch nur durch seine Form, wie Einfassungen der Gemälde oder Gewänder an Statuen, oder Säulengänge um Prachtgebäude (KANT 1972: 65).
\end{abstract}

Was Herder nach der Logik eines in die Imagination des lebendigen Gefühls nicht integrierbaren Widerstands problematisiert und als Unterbrechung des rezeptiven Betrugs beschreibt, nämlich Gewänder, fasst Kant als ornamentalen ,Zusatz' zusammen. Diesen setzt er als Nebenwerk, Parergon, dem als, inneres' Kondensat verstandenen Werk entgegen wie Herder die Verhüllung dem Körper und mit ihr die leblose Materie der imaginären Verlebendigung. Indem er jedoch die Paradoxie der nicht verhüllenden bzw. adverbial in ihrer Bedeutung zurückgenommenen, gleichsam'-Kleider entwirft, denkt er eine Verbindung beider Seiten - die auch Kant im Bezug auf die ,Form' hervorhebt - mit. Grundsätzlich gehören nach Herder Kleider und Gewänder der internen Logik der Kunst nicht an. Den ,nassen Gewändern“ wird der Kunststatus ${ }^{7}$ hingegen nicht gänzlich abgesprochen, sofern sie nämlich den Körper - ,das Wesen der Kunst ${ }^{6}$ - nicht verdecken, sondern vielmehr durchscheinen lassen und zugänglich machen. ${ }^{8}$ Solche Gewänder

7 Wichtig ist hierbei, dass Herder diese Gewänder als eine kunstimmanente Technik bewertet. Dagegen vermutete Winckelmann in ihnen, wie Herder hier rekapituliert, die realen Gewänder der Griechen und setzte sie so in ein Abbildverhältnis zur gesellschaftlichen Realität.

8 Jacques Derrida kritisiert Kants Unterscheidung zwischen Werk und Bei- oder Nebenwerk an einer Stelle, indem er nach den Grenzen des Parergons fragt und sie anhand der Gewänder thematisiert: „Das Vorgestellte der Vorstellung wäre 
dürfen formal von den Proportionen und Konturen des Körpers, der durch sie hindurch erst hervortreten soll, nicht abweichen. Sie müssen sich ihm vollständig fügen, seiner Gestalt sich unterordnen, sie gleichsam, wie eine zweite Haut, modellieren. Damit sie zumindest zum Rand bzw. zur Schwelle des Kunstwerks selbst vordringen können, um - mit Kant gesprochen - ,das Wohlgefallen des Geschmacks zu vergrößern', dürfen sie kein von dem ,eigentlichen' Gegenstand der Skulptur unterschiedenes Eigenleben entfalten. ${ }^{9}$

Besteht aber der Zierat nicht selbst in der schönen Form, ist er, wie der goldene Rahmen, bloß, um durch seinen Reiz das Gemälde dem

der nackte und natürliche Körper; das vorstellungsmäßige Wesen der Statue bezöge sich darauf, und, an sich, wäre allein diese Vorstellung schön, wesentlich, rein und intrinsisch schön, eigentlicher Gegenstand eines reinen Geschmacksurteils.“ Im neuen Absatz fährt Derrida fort: „Diese Abgrenzung des Zentrums und der Gesamtheit der Vorstellung, ihres Innen und ihres Außen, mag schon ungewöhnlich scheinen. Man fragt sich überdies, wo man die Bekleidung anfangen lassen soll. Wo beginnt und wo endet ein Parergon? Wäre jede Bekleidung ein Parergon. Die Slips und anderes. Was macht man mit völlig durchsichtigen Schleiern. [...] Es ist die Frage nach dem vorstellungsmäßigen und vergegenständlichenden Wesen, nach seinem Innen und seinem Außen, nach den Kriterien, die für diese Abgrenzung in Anspruch genommen werden, nach dem Natürlichkeitswert, der hierbei vorausgesetzt wird, und, nebensächlich oder wesentlich, nach dem Platz des menschlichen Körpers oder seinem Privileg in dieser ganzen Problematik.“ (DERRIDA 1992: 77).

9 Herder selbst spricht aber in einem anderen Zusammenhang von Parerga: wenn er nämlich von Körpern von Tieren, Schlangen, Schildkröten, spricht. „Niemand wird's in den Sinn kommen, solche Geschöpfe für das Hauptwerk der Kunst zu halten: der Mensch thront auf ihrem Altar, ihm ist die Bildsäule heilig. Aber nun, als Beigerät, als Nebenwerk, als Fußschemel, welcher Tor darf da verbieten und untersagen“ (HERDER: 273). Dieser Nebenort kann zugleich für die Aufnahme des Hässlichen genutzt werden. Dabei sind hier auch Darstellungsmöglichkeiten erlaubt, die das eigentliche Werk nicht zulassen darf. Dieses der Skulptur zugeordnete ,Beigerät ${ }^{`}$ ist derart lose angebunden, dass es sich entfernen und ablösen ließe, ohne das Werk selbst zu gefährden. Die Abgrenzung zwischen dem ,Hauptwerk ${ }^{\varsigma}$ und dem ,Beigerät ${ }^{\star}$ steht in diesem Fall nicht zur Disposition. 
Beifall zu empfehlen, angebracht, so heißt er alsdann Schmuck und tut der echten Schönheit Abbruch (KANT 1972: 65).

Der hier angesprochene, Abbruch der echten Schönheit' hat denselben strukturellen Ort wie die Störung der ästhetischen Rezeption im Widerstand des ,Steinklumpens' bei Herder. In beiden Fällen wird dabei nicht nur zwischen dem Werk und seinem Außen, sondern auch innerhalb dieses selbst jeweils eine Grenze gezogen. Unterschiedliche Typen des Werkaußen, unterschiedliche Distanzstufen werden vermessen, um ein Parergon, welches dem Werk dient, es somit stützt, ja sich ihm gleichsam‘ anverwandelt, von einem Parergon abzugrenzen, welches wie das ,Unding', der ,Steinklumpen“ oder bloßer ,Schmuck' es beeinträchtigt. Eine maximale Annäherung des Beiwerks an das Werk ist in den ,nassen Gewändern', wie Herder sie beschreibt, gegeben. Nur eine hauchdünne Schicht trennt hier die beiden Bereiche, die Darstellung des Körpers und seine Verhüllung, voneinander. Mag nun das Parergon wie bei den ,nassen Gewändern' kaum wahrnehmbar sein, so ist es zugleich unerlässlich. Genau diesen Aspekt pointiert Derrida in seiner KantLektüre. ,Was sie zu Parerga macht, ist nicht einfach ihre überflüssige Äußerlichkeit, es ist das interne strukturelle Band, das sie mit dem Mangel im Inneren des Ergon zusammenschweißt. “"10 Den konstitutiven Beitrag am Werk muss es aber invisibilisieren. Dem ästhetischen Anspruch, das Beiwerk aus dem Werk auszuschließen, korreliert eine entgegengesetzte Problemlage, insofern das Werk von seinem Beiwerk abhängig ist. Durch einen, innerlichen Mangel' bestimmt, braucht das Werk exakt jene Elemente, von welchen es sich als bloßen Äußerlichkeiten und Zusätzen zugleich distanziert.

Herder behandelt die Parergon-Problematik in der Bildhauerkunst der griechischen Antike jedoch als einen Sonderfall: ,Wo es ihm [dem Griechen] ein Gesetz auflegte', heißt es da, also nicht immer. Er räumt damit einen Normalfall ein, demzufolge eine uneingeschränkte Realisation des ästhetischen Maßstabs und damit die Anschauung eines parergonfreien Ergon vorherrscht. Welche Exempla aber legt er für diese vollkommene Plastik dar? Wenn er die Aufgabe der Bildhauerei als Bildung ,einer Gestalt

10 DERRIDA 1992: 80. 
der Schönheit, der Liebe, des Reizes, der Jugend, 'bezeichnet, dann nennt er hier abstrakte Begriffe, die Göttern zugeschrieben und auf eine Darstellung festgelegt werden, die nicht nur auf Gewänder zu verzichten hat, sondern sich auch von anderen, dem nackten Körper angehängten Attributen freihalten soll. In diesem Sinne ergänzt er die genannte Reihe: „Die Göttin der Liebe ohne drückende Attribute: sie selbst ist Göttin der Liebe, in nackte Reize gekleidet" (277). Bezeichnend ist an dieser Formulierung, dass die Kleidung hier als Metapher auftritt, die der eigentlichen Bestimmung nicht entgegengesetzt wird, sondern sie vielmehr bestätigt. Das kursive, sie selbst', dem die ,nackten Reize' entsprechen, verbindet sich mit exakt demjenigen Element, welches das Parergon einer Skulptur repräsentiert, der Kleidung, jedoch nicht, um es mittels eines ,Gleichsam` zum Werk auf Distanz zu halten. Denn die ,nackten Reize` der Venus sind ihr ,Kleid‘. So wird zumindest rhetorisch die für die Argumentation des Textes ausschlaggebende Differenz zwischen dem Werk und seinem Beiwerk aufgehoben.

Wie aber und anhand welcher Beispiele, um die Frage erneut zu stellen, wird in der „Plastik“ ein parergonfreies Werk beschrieben? Im zweiten Teil des zweiten Kapitels wendet sich Herder eben dieser Problematik zu. Nachdem er die Kleider und Gewänder als Beiwerk reflektiert und nur im Fall der ,nassen Gewänder' der Bildhauerei zugestanden hat, lotet er nun den Bereich dessen aus, was den ,eigentlichen' Gegenstand dieser Kunstform ausmacht. „Viel feinere Sachen, “schränkt Herder jedoch auch in bezug auf den schönen menschlichen Körper ein,

müssen von der Statue wegbleiben, weil sie dem Gefübl widerstehen, weil sie dem tastenden Sinn keine ununterbrochene schöne Form sind. Diese Adern an Händen, diese Knorpeln an Fingern, diese Knöchel an Knien müssen so geschont, und in Fülle des Ganzen verkleidet werden; oder die Adern sind kriechende Würme, die Knorpel aufliegende Gewächse dem stillen dunkeltastenden Gefühl. Nicht ganz Fülle Eines Körpers mehr, sondern Abtrennungen, losgelöste Stücke des Körpers, die seine Zerstörung weissagen, und sich daher schon selbst entfernten (269).

Zwei auf dem Feld der Anthropologie fußende Funktionsbereiche werden hier miteinander konfrontiert. Einerseits wird auf das von der $\mathrm{Na}-$ 
turforschung des späten 18. Jahrhunderts entwickelte Organismuskonzept, andererseits auf die ästhetische Forderung einer ,schönen Form'verwiesen. Der Konflikt besteht dabei darin, dass der organische Zusammenhang nicht nur die ,Fülle eines Körpers', sondern auch dessen ,Zerstörung' und Vergänglichkeit einbezieht. ${ }^{11}$

Wenn Leben als organischer Prozess definiert wird, dann ist ihm, wie Herder in den ,Abtrennungen' und ,losgelösten Stücken des Körpers' hervorhebt, sein eigenes Absterben bereits eingeschrieben. Die von Herder entworfene Ästhetik der Bildhauerei ist hingegen ausschließlich dem Ausdruck der Vitalität ${ }^{12}$ verpflichtet. In der ästhetischen Applikation wird der organische Körper somit auf die Dimension des sich erhaltenden Lebens beschränkt. Die andere, es bedrohende Seite muss die Darstellung daher verdecken; ein Vorgang, für welchen die vestimentäre Metaphorik bemüht wird. So spricht der Text ausdrücklich von, Verkleidung', um die ,Adern an Händen, diese Knorpeln an Fingern, diese Knöchel an Knien' unsichtbar zu machen. Es handelt hier nicht nur um einen rhetorischen Verweis. Vielmehr wird die Operation der, Verkleidung' selbst auf die skulpturale Darstellung des menschlichen Körpers übertragen. Herder optiert für eine Verhüllung jener physiologischen Elemente, an welchen sich die Zerstörung des menschlichen Körpers ablesen lässt. Nur „,die blauen Adern unter der Haut“ dürfen sichtbar sein, denn ,sie duften Leben, da wallet Blut; als Knorpel und Knochen sind sie nur fühlbar und haben kein Blut und duften kein Leben mehr, in ihnen schleicht der lebendige Tod“" (269).

Die Beschreibung des Gebeins - der ,Knorpel und Knochen - knüpft an die allegorische Tradition des Todesinszenierung an, während die Bezugnahme auf das Leben als, unter der Haut' verlaufende Adern die phy-

11 Vgl. Foucault.

12 „Der simple Satz war meine Absicht: ,daß jede Form der Erhabenheit und Schönheit am menschlichen Körper eigentlich nur Form der Gesundheit, des Lebens, der Kraft, des Woblseins in jedem Gliede dieses kunstvollen Geschöpfes, so wie hingegen Alles Häßliche nur Krüppel, Druck des Geistes, unvollkommene Form zu ihrem Endzweck sei und bleibe“ (296). Bzw. anders: „Schönheit ist also nur immer Durchschein, Form, sinnlicher Ausdruck der Vollkommenheit zum Zwecke, wallendes Leben, Menschliche Gesundheit“ (297). 
siologische Einheit des Blutkreislaufs konnotiert. Entscheidend ist an dieser Beschreibung aber, dass sie das organische Bild, unter die Haut' legt. Es wird daher in einer Weise bedeckt gehalten, die an die ,nassen Gewänder ${ }^{6}$ erinnert. Die Adern dürfen zum Vorschein kommen, sofern sie sich als Teil der Blutzirkulation zu erkennen geben, die Knochen und Knorpel - Sinnbilder des Todes - müssen hingegen gänzlich unter der Haut verschwinden. Aber auch bestimmte physiognomische Merkmale, die ein Gleiten des ,fühlenden Fingers' stören könnten, weil sie die Regelmäßigkeit der Haut unterbrechen, sind zurückzudrängen. Die Haut wird zu einem Regulator dessen, was sichtbar werden darf, und dessen, was es zu verdecken gilt, weshalb sie sowohl transparent als auch opak gedacht werden muss. Eine Ökonomie des Sichtbaren, die unter Berufung auf das Gefühl legitimiert wird. Der gegenseitigen Abhängigkeit von Sehen und Tasten, Malerei und Plastik vergleichbar, fällt auch hier die Distinktion zwischen Werk und Beiwerk zusammen. Hat Herder nämlich die Funktion der, nassen Gewänder ' zunächst so beschrieben, als schmiegten sie sich wie eine zweite Haut an den Körper an, wird hier umgekehrt die Haut mit der Funktion der Kleidung identifiziert. Somit wird das Parergon in zweifacher Hinsicht zum Bestandteil des Ergon; es verwandelt sich ihm, gleichsam' an oder es tritt an dessen Stelle.

\section{Literaturverzeichnis}

Foucault, Michel. Die Geburt der Klinik. Eine Archäologie des ärz̨tlichen Blicks. Übers. von Walter Seitter. Frankfurt a. M. 1988.

Derrida, Jacques. Die Wabrheit in der Malerei. Übers. von Michael Wetzel. Wien 1992.

Genette, Gérard. Paratexte. Das Buch vom Beiwerk des Buches. Übers. von Dieter Hornig. Frankfurt a. M. / New York 1992.

KanT, Immanuel. Kritike der Urteilskraft. Hg. von Karl Vorländer. Hamburg 1974.

Herder, Johann Gottfried. „Plastik. Einige Wahrnehmungen über Form und Gestalt aus Pygmalions Träume“. In: Ders., Werke in zehn Bänden, Bd. 4: Schriften zu Philosophie, Literatur und Altertum 1774-1787. Hg. von Jürgen Brummack und Martin Bollacher. Frankfurt a. M. 1994. 